\title{
Information and Energy Flows in Graphical Networks with Energy Transfer and Reuse
}

\author{
Ali Mohammad Fouladgar and Osvaldo Simeone, Member, IEEE
}

\begin{abstract}
The conventional analysis of communication over graphical networks assumes that the energy received by a node on its incoming links cannot be reused by the latter for transmission on its outgoing links. Motivated by the emerging applications of wireless energy transfer to communication networks, a framework is studied in which the received energy can be (partially) reused. The framework leads to optimization problems over both standard information flow variables and energy flow variables that generalize the conventional formulation in terms of information flow variables only. Both unicast and multicast models are studied, and numerical results are presented that bring insight into the roles of the energy flow variables and of network coding in the presence of energy reuse.
\end{abstract}

Index Terms-Information and energy transfer, energy harvesting, routing, network coding, graphical networks.

\section{INTRODUCTION}

$\mathbf{T}$ HE conventional design of communication networks is based on the assumption that the energy received by a node cannot be reused for later communication tasks. However, a number of applications challenge this approach, including RFID systems [1] and body area networks [2]. The enabling technology behind these applications is wireless energy transfer, which has a long history [3] and is on its way to become widely available commercially (see, e.g., [4][5]). It is noted that the efficiency of wireless energy transfer via nearfield induction can be as high as $90 \%$ [4], whereas that of farfield radiations is generally much lower, depending critically on the antenna sizes and the communication distance, but is sufficient to warrant its commercial viability [5].

Based on the observations above, a number of works have recently tackled the analysis of communication systems under the assumption that the received energy can be reused. Specifically, previous work [6], [7] has focused on the problem of maximizing the information rate of single-source singlehop systems subject to minimum average received energy constraints. Reference [6] studied a single point-to-point channel, while [7] investigated a set of parallel point-to-point channels. The optimization of beamforming strategies for multiantenna broadcast channels under a similar criterion has been studied in [8].

All the works mentioned above consider single-hop networks. In [9], interactive channels with two nodes were studied in the presence of energy reuse. In this paper, instead, we tackle the analysis of general multi-hop networks with energy

Manuscript received January 13, 2013. The associate editor coordinating the review of this letter and approving it for publication was H. Jiang.

The authors are with the Center for Wireless Communications and Signal Processing Research (CWCSPR), ECE Department, New Jersey Institute of Technology (NJIT), Newark, NJ 07102, USA (e-mail: \{af82, osvaldo.simeone\}@njit.edu).

Digital Object Identifier 10.1109/WCL.2013.042313.130029 reuse. We adopt standard graphical network models (see, e.g., [10]) and assume that the energy received by a node on its incoming links can be reused by the latter for transmission on its outgoing links. The analysis leads to (convex) optimization problems over both standard information flow variables and energy flow variables that generalize the conventional formulation in terms of information flow variables only (see [10][11]). We treat the unicast case in Sec. II and the multicast set-up in Sec. III. In both cases, we also provide numerical results that bring insight into the roles of the energy flow variables and of network coding in the presence of energy reuse. We refer to [12] for a feasibility study of wireless energy transfer across multiple hops.

\section{UNICAST NETWORK}

Consider a standard unicast network modeled by a graph $\mathcal{G}=(\mathcal{N}, \mathcal{E})$. The set of nodes is $\mathcal{N}=[1: N]$ and $\mathcal{E} \subseteq \mathcal{N} \times \mathcal{N}$ is the set of edges. Source node 1 wishes to communicate a message $M \in\left[1: 2^{n R}\right]$ to the single destination node $N$. Each node $k \in[2: N-1]$ acts as a relay to help the source node communicate its message to the destination node $N$. An example is shown in Fig. 1. The extension to multiple sources, each with its own destination (the destinations are not necessarily distinct), is straightforward and will be briefly discussed below.

Each edge $(j, k)$ between node $j$ and node $k$ is characterized by a function $f_{j k}\left(r_{j k}\right)$ that provides the average energy necessary to transmit at an information rate equal to $r_{j k} \geq 0$ on the $(j, k)$ link. Following conventional definitions (see, e.g., [13]), we refer to the variables $\left\{r_{j k}\right\}$ as specifying the information flow in the network. We assume that function $f_{j k}\left(r_{j k}\right)$ is monotonically increasing and convex. An example is Shannon's optimal energy-rate function for Gaussian channels, i.e.,

$$
f_{j k}\left(r_{j k}\right)=\frac{2^{r_{j k}}-1}{g_{j k}},
$$

where $g_{j k}$ represents the channel energy gain for the $(j, k)$ link assuming unit-power noise (recall that $r_{j k}=\log _{2}(1+$ $\left.g_{j k} f_{j k}\left(r_{j k}\right)\right)$ ). We denote as $p_{j k}$ the average energy used for transmission on the $(j, k)$ link. The average energies $\left\{p_{j k}\right\}$ are defined as the energy flow variables. From the discussion above, we have the inequality

$$
p_{j k} \geq f_{j k}\left(r_{j k}\right)
$$

since $f_{j k}\left(r_{j k}\right)$ is the energy required to transmit at rate $r_{j k}$ on link $(j, k)$.

In the conventional model in which the nodes cannot collect and reuse the received energy, it is clearly optimal to have (2) satisfied with equality. In fact, any additional energy in excess 


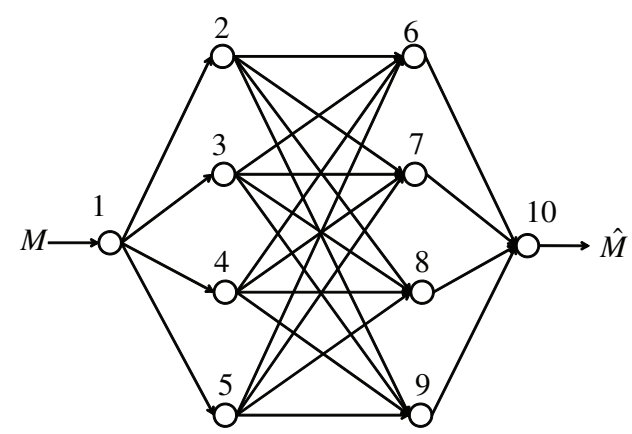

Fig. 1. An example of a graphical unicast network.

of $f_{j k}\left(r_{j k}\right)$ would not contribute to the system performance. However, this is not the case when nodes can harvest energy from the received signal for reuse on their outgoing links. In order to clearly identify the requirements of information and energy transfer, we observe that the average energy

$$
p_{j k}^{e}=p_{j k}-f_{j k}\left(r_{j k}\right)
$$

is used solely for energy transfer, since the energy needed for information transfer at a rate $r_{j k}$ is $f_{j k}\left(r_{j k}\right)$. We refer to $p_{j k}^{e}$ as the excess energy flow on link $(j, k)$. Given the energy flow variables $\left\{p_{j k}\right\}$, the average energy harvested by the $k$ th node is given by

$$
q_{k}=\sum_{j:(j, k) \in \mathcal{E}} \alpha_{j k} p_{j k}+\beta_{k}
$$

In (4), the parameters $\alpha_{j k}$ account for path loss on the $(j, k)$ link and for the efficiency of energy transfer and storage at node $k$, while $\beta_{k}$ is the average energy obtained by node $k$ from external sources such as the electrical grid or solar panels.

\section{A. Maximum Rate}

In this section, we characterize the maximum rate $R$ at which the source 1 can communicate reliably to the destination $N$ on the network described above. We first observe that the information flow variables must satisfy the conventional information flow conservation conditions (see (6d)-(6e) below). Note that an implicit assumption behind this standard formulation is that the nodes have sufficiently long data buffers. As far as the energy flow is concerned, we instead need to impose the energy balance conditions

$$
q_{k} \geq \sum_{l:(k, l) \in \mathcal{E}} p_{k l}
$$

to all nodes $k \in \mathcal{N}$. Constraint (5) states that the total average incoming energy $q_{k}$ in (4) at each $k$ th node should be larger than the total outgoing energy from it, which is given by the right-hand side of (5). The fact that the constraint (5) is sufficient to ensure energy conservation at node $k$ holds under the assumption that the nodes have sufficiently long energy buffers (see, e.g., [14][15]).

Overall, the maximum rate $R$ and corresponding optimal information and energy flow variables, namely $r_{j k}$ and $p_{j k}$ for all edges $(j, k) \in \mathcal{E}$, respectively, can be found by solving the following optimization problem:

$$
\begin{array}{ll}
\text { maximize } & R \\
\text { subject to } & R \geq 0, r_{j k} \geq 0, p_{j k} \geq 0 \text { for all }(j, k) \in \mathcal{E} \\
& p_{j k} \geq f_{j k}\left(r_{j k}\right) \text { for all }(j, k) \in \mathcal{E} \\
& \sum_{j:(j, k) \in \mathcal{E}} r_{j k}=\sum_{l:(k, l) \in \mathcal{E}} r_{k l} \text { for all } k \in \mathcal{N} \backslash\{1, N\} \\
& \sum_{k:(1, k)} r_{1 k}=R, \sum_{j:(j, N)} r_{j N}=R \\
& \sum_{j:(j, k) \in \mathcal{E}} \alpha_{j k} p_{j k}+\beta_{k}-\sum_{l:(k, l) \in \mathcal{E}} p_{k l} \geq 0 \text { for all } k \in \mathcal{N},
\end{array}
$$

where we recall that (6f) represents the energy conservation condition (5) due to (4). We observe that the problem (6) is convex, due to the convexity of functions $f_{j k}\left(r_{j k}\right)$, and thus can be solved using standard tools (see, e.g., [16]).

A few further remarks are in place. First, by assuming that the energies $p_{j k}$ are fixed, constraint (6c) reduces to the standard per-link capacity constraint. Therefore, when eliminating the energy conservation constraint (6f), fixing the energies $p_{j k}$ leads to the standard maximum information flow problem over the variables $\left\{r_{j k}\right\}$ (see, e.g., [10, Ch. 15]). As a second remark, we observe that the problem formulation (6) can be easily extended to the case in which there are multiple sources nodes, each with its own destinations (the destinations are not necessarily distinct). This can be done by introducing an information flow variable $r_{m, j k}$ for each $m$ th sourcedestination pair and each link $(j, k) \in \mathcal{E}$. The information flow conditions (6d)-(6e) are then imposed separately for each $m$, while (6c) and (6d) remain unchanged with the definition $r_{j k}=\sum_{m} r_{m, j k}$ for the total information flow on link $(j, k)$.

\section{B. Numerical Results}

Consider the graphical unicast wireless fading network illustrated in Figure 1 in which source 1 communicates to destination 10 through two layers of four relay nodes each. For each link, we assume the energy-rate function (1), where the channel energy gains $g_{j k}$ are independent and distributed as unit-power exponential variables (Rayleigh fading). We also set $\alpha_{j k}=0.5$ for all links $(j, k) \in \mathcal{E}$ in (4). Finally the average energy $\beta_{k}$ obtained by node $k$ from external sources in (4) is $\beta_{k}=0.1$ for all relay nodes $k \in[2: 9]$, while $\beta_{1}$ is varied for the source node.

In Fig. 2, we show the rate $R$ obtained by solving problem (6), as averaged over the realization of the channel gains $\left\{g_{j k}\right\}$. For reference, we consider two strategies. In the first, we constrain the network to select only the rate-maximizing path between source and destination, where a path consists of the links $(1, j),(j, k),(k, 10)$ with $j \in\{2,3,4,5\}$ being a relay in the first layer and $k \in\{6,7,8,9\}$ being a relay in the second layer of the network in Fig. 1. The second reference strategy is such that it sets the excess energy flow variables $p_{j k}^{e}$ to zero for all the outgoing links. As a result, this strategy uses on each link only the minimal energy necessary to support the information flow on the link, without allowing for additional energy transfer to be used on downstream links towards the 


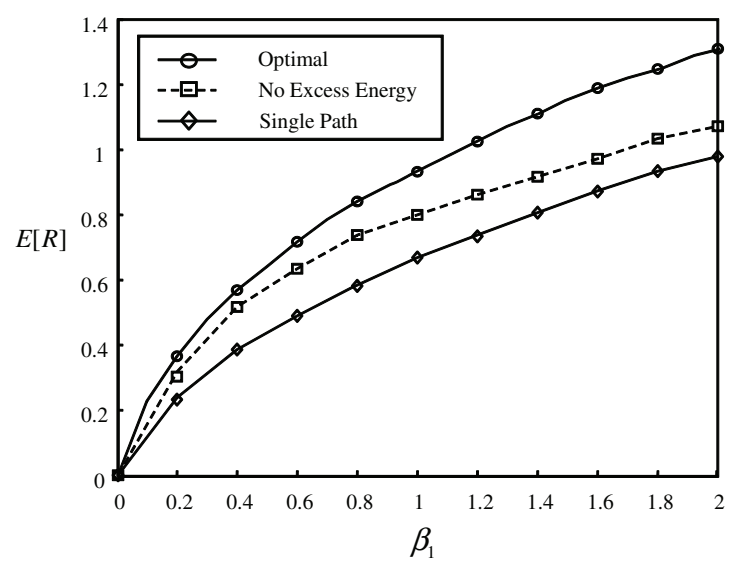

Fig. 2. Average rate $E[R]$ versus the exogenous energy level $\beta_{1}$ at the source node 1 for the unicast network of Fig. $1\left(\alpha_{j k}=0.5, \beta_{k}=0.1\right.$ for $(j, k) \in \mathcal{E}$ and $k \in[2: 9])$.

destination. The rate for this strategy is obtained by imposed that the constraint $(6 \mathrm{c})$ be satisfied with equality. ${ }^{1}$

In Fig. 2, the average rates $R$ for the optimal strategy and for the two references methods discussed above are shown versus the exogenous energy level $\beta_{1}$ at the source node 1 . The performance loss of not allowing for an excess energy flow is apparent and is increasing with the exogenous energy $\beta_{1}$ available at the source. This is because more energy at the source enables a more effective management of the energy flow in the network if non-zero excess energies are allowed. Moreover, it is seen that forcing the network to use only a single path leads to a significant rate degradation. This suggests that both the ability to route information and energy over multiple paths and the use of excess energy flows are instrumental in obtaining the optimal performance.

\section{Multicast Network}

In this section, we study a multicast network, modeled by a directed graph $\mathcal{G}=(\mathcal{N}, \mathcal{E})$. Unlike the unicast case studied in Sec. II, source node 1 wishes to communicate a message $M \in\left[1: 2^{n R}\right]$ to a set of destination nodes $\mathcal{D} \subset \mathcal{N} \backslash\{1\}$. An example is shown in Fig. 3. All other definitions are as in Sec. II.

\section{A. Maximum Rate}

We investigate the maximum multicast rate $R$ at which node 1 can communicate to the set of destinations $\mathcal{D} \subset \mathcal{N} \backslash\{1\}$. As well known, for any given capacity, and thus energy, allocation on all links, the maximum rate is achieved by network coding [13]. For reference, we first consider the performance achievable with routing.

\footnotetext{
${ }^{1}$ Note that this equality makes problem (6) non-convex. To deal with this issue, first, by using the constraint $p_{j k}=f_{j k}\left(r_{j k}\right)$, problem (6) can be reformulated with respect to the information flow variables $\left\{r_{j k}\right\}$ only. The resulting (non-convex) optimization is a difference-of-convex problem [16], for which stationary points can be easily obtained via the MajorizationMaximization (MM) algorithm [16].
}

1) Routing: To enable routing, we follow the standard approach (see, e.g., [17]) and split the message $M$ into $n$ submessages $M_{m}$, with $m \in[1: n]$ as $M=\left(M_{1}, M_{2}, . ., M_{n}\right)$, where the $m$ th submessage $M_{m} \in\left[1: 2^{n R_{m}}\right]$ has rate $R_{m}$. Each message $M_{m}$ is routed on a tree that is rooted at node 1 and whose leaves are the destination nodes $\mathcal{D}$. The trees are described by defining variables $a_{m, j k}$ for all $m \in[1: n]$ and $(j, k) \in \mathcal{E}$ as follows: $a_{m, j k}=1$ if the link $(j, k)$ belongs to the tree of message $M_{m}$ and otherwise $a_{m, j k}=0$.

The maximum rate $R$ achievable by routing and the corresponding optimal information and energy flow variables $r_{j k}$ and $p_{j k}$ for all edges $(j, k) \in \mathcal{E}$, can then be found by solving the following optimization problem:

$$
\begin{array}{ll}
\text { maximize } & \sum_{m=1}^{n} R_{m} \\
\text { subject to } & R_{m} \geq 0 \text { for all } m \in[1: n] \\
& r_{j k} \geq 0, p_{j k} \geq 0 \text { for all }(j, k) \in \mathcal{E} \\
& p_{j k} \geq f_{j k}\left(r_{j k}\right) \quad \text { for all }(j, k) \in \mathcal{E} \\
& \sum_{m=1}^{n} a_{m, j k} R_{m}=r_{j k} \text { for all }(j, k) \in \mathcal{E} \\
& \sum_{j:(j, k) \in \mathcal{E}} \alpha_{j k} p_{j k}+\beta_{k}-\sum_{l:(k, l) \in \mathcal{E}} p_{k l} \geq 0 \text { for all } k \in \mathcal{N} .
\end{array}
$$

Condition (7e) states that the sum of all the information flows passing through a link $(j, k)$ cannot be larger than the overall energy flow $r_{j k}$. Instead, the inequality (7f) imposes energy conservation at each node $k$ as in (6f). Similar to problem (6), the optimization (7) is also a convex problem and hence can be solved efficiently [16].

2) Network Coding: Here, we characterize the maximum rate $R$ achievable using network coding. Following [11], we introduce an information flow variable $r_{j k}^{(d)}$ for each destination $d \in \mathcal{D}$ and each edge $(j, k) \in \mathcal{E}$. The maximum rate $R$ achievable via network coding and the corresponding optimal information flows variables $r_{j k}^{(d)}$ and energy flow variables $p_{j k}$ for all edges $(j, k) \in \mathcal{E}$, can then be found by solving the following optimization problem:

$$
\begin{array}{ll}
\text { maximize } & R \\
\text { subject to } & R \geq 0, r_{j k}^{(d)} \geq 0, p_{j k} \geq 0, r_{j k} \geq 0, \\
& \text { for all }(j, k) \in \mathcal{E} \text { and } d \in D \\
& r_{j k}^{(d)} \leq r_{j k} \text { for all }(j, k) \in \mathcal{E} \text { and } d \in \mathcal{D} \\
& p_{j k} \geq f_{j k}\left(r_{j k}\right) \quad \text { for all }(j, k) \in \mathcal{E} \\
& \sum_{j:(j, k) \in \mathcal{E}}^{(8 \mathrm{c})} r_{j k}^{(d)}=\sum_{l:(k, l) \in \mathcal{E}} r_{k l}^{(d)} \\
& \text { for all } k \neq 1, N, \text { and } d \in \mathcal{D} \\
& \sum_{k:(1, k)} r_{1 k}^{(d)}=R, \sum_{j:(j, N)} r_{j N}^{(d)}=R, \text { for all } d \in \mathcal{D} \quad(8 \mathrm{~d}) \\
& \sum_{j:(j, k) \in \mathcal{E}} \alpha_{j k} p_{j k}+\beta_{k}-\sum_{l:(k, l) \in \mathcal{E}} p_{k l} \geq 0 \text { for all } k \in \mathcal{N} .(8 \mathrm{~g})
\end{array}
$$

Similar to (6d)-(6e), the conditions (8e)-(8f) impose the information flow constraints for each destination $d \in \mathcal{D}$, and inequality $(8 \mathrm{~g})$, as (6f), ensures energy conservation. Constraints (8c) and (8d) follows from the results in [11]. Specifically, as 


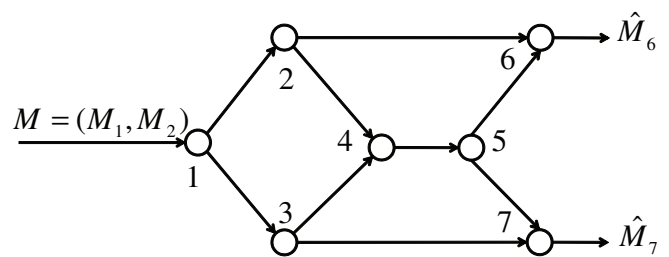

Fig. 3. An example of a multicast graphical network with destination set $\mathcal{D}=\{6,7\}$.

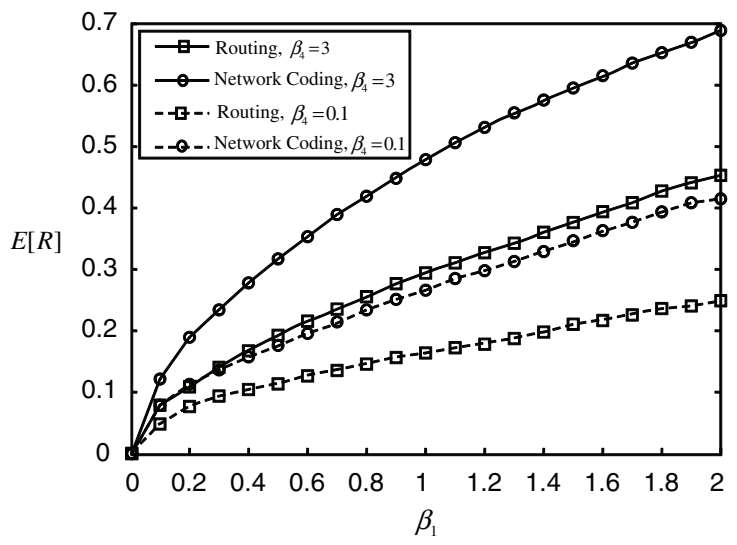

Fig. 4. Average rate $E[R]$ versus the exogenous energy level $\beta_{1}$ at the source node 1 for the multicast network of Fig. 3 ( $\alpha_{j k}=0.5, \beta_{k}=0.1$ for $(j, k) \in \mathcal{E}$ and $k \in[2: 7] \backslash\{5\})$.

shown in [11], based on [13], the overall information flow $r_{j k}$ for each link $(j, k)$ is equal to

$$
r_{j k}=\max _{d \in \mathcal{D}} r_{j k}^{(d)}
$$

The fact that the maximum rather than the sum of the information flows, as in routing (cf. (7e)), affects the performance is a consequence of the achievability of the cut-set bound by network coding [13]. Condition (8c) then imposes that the information flow variables $\left\{r_{j k}\right\}$ and $\left\{r_{j k}^{(d)}\right\}$ satisfy the condition (9). Problem (8) is convex and can be solved via standard tools [16].

\section{B. Numerical Results}

Consider the butterfly multicast network illustrated in Figure 3 with $\mathcal{D}=\{6,7\}$. For each link, like Section II-B, we assume the energy-rate function (1), where the channel energy gains $g_{j k}$ are independent and distributed as a unit-power exponential variables (Rayleigh fading). We also set $\alpha_{j k}=0.5$ for all links $(j, k) \in \mathcal{E}$, and $\beta_{k}=0.1$ for all relay nodes except node 1 and node 4 . We solve the optimization problems (7) and (8) in order to evaluate the system performance with network routing and network coding, respectively. Specifically, for routing, we split the message $M$ into $n=2$ submessages $M_{1}$ and $M_{2}$, where $M_{m} \in\left[1: 2^{n R_{m}}\right]$ for $m=1,2$, so that $R_{m}$ is the rate of message $M_{m}$. The tree for message $M_{1}$ consists of edges $(1,2),(2,4),(2,6),(4,5),(5,7)$ and the tree for message $M_{2}$ consists of edges $(1,3),(3,4),(3,7),(4,5),(5,6)$.
Figure 4 shows the average rates achievable with routing and network coding versus the exogenous energy level $\beta_{1}$ at the source node 1 for two values of the exogenous energy level at node 4 . We observe that, for routing, the link $(4,5)$ belongs to the trees of both submessages, and hence the transmitted energy of node 4 must support the sum-rate $R_{1}+R_{2}$. Network coding instead alleviates this problem by coding across the two streams [13]. As a result, Figure 4 demonstrates the significant gains of network coding over routing. Moreover, this performance gain increases as the energy $\beta_{4}$ grows larger due to enhanced capability of network coding to leverage the energy at node 4 thanks to coding.

\section{Conclusions}

The emerging paradigm of communication networks powered by the received radio signal calls for a revision of conventional analyses and design approaches. In this paper, we have taken a first step in this direction for the investigation of multihop networks. The presented analysis focuses on graphical networks and reveals the roles of energy flow variables and network coding in the presence of energy reuse. Extensions of interest include the design of decentralized solutions, using, e.g., dual decomposition as in [11].

\section{REFERENCES}

[1] V. Chawla and S. H. Dong, "An overview of passive RFID," IEEE Commun. Mag., vol. 45, no. 9, pp. 11-17, Sep. 2007.

[2] F. Zhang, X. Liu, H. Chen, R. J. Sclabassi, and M. Sun, "Wireless energy transfer platform for medical sensors and implantable devices," in Proc. 2009 Int. Conf. IEEE Engineering in Medicine and Biology Society, pp. 1045-1048.

[3] W. C. Brown, "The history of power transmission by radio waves," IEEE Trans. Microwave Theory and Techniques, vol. 32, no. 9, pp. 1230-1242, Sept. 1984.

[4] http://www.wirelesspowerconsortium.com/

[5] http://www.powercastco.com/

[6] L. R. Varshney, "Transporting information and energy simultaneously," in Proc. 2008 IEEE Int. Symp. Inform. Theory, pp. 1612-1616.

[7] P. Grover and A. Sahai, "Shannon meets Tesla: wireless information and power transfer," in Proc. 2010 IEEE Int. Symp. Inform. Theory, pp. 2363-2367.

[8] R. Zhang and C. K. Ho, "MIMO broadcasting for simultaneous wireless information and power transfer," arXiv:1105.4999.

[9] P. Popovski, A. M. Fouladgar, and O. Simeone, "Interactive joint transfer of energy and information," to appear in IEEE Trans. Commun.

[10] A. El Gamal, and Y.-H. Kim, Network Information Theory. Cambridge University Press, 2011.

[11] D. S. Lun, M. Medard, T. Ho, and R. Koetter, "Network coding with a cost criterion," in Proc. 2004 IEEE Int. Symp. Inform. Theory.

[12] M. K. Watfa, H. AlHassanieh, and S. Selman, "Multi-hop wireless energy transfer in WSNs," IEEE Commun. Lett., vol. 15, no. 12, pp. 1275-1277, Dec. 2011.

[13] R. Ahlswede, N. Cai, S.-Y. R. Li, and R. W. Yeung, "Network information flow," IEEE Trans. Inf. Theory, vol. 46, no. 4, pp. 1204-1216, Jul. 2000.

[14] V. Sharma, U. Mukherji, V. Joseph, and S. Gupta, "Optimal energy management policies for energy harvesting sensor nodes," IEEE Trans. Wireless Commun., vol. 9, no. 4, pp. 1326-1336, Apr. 2010.

[15] O. Ozel and S. Ulukus, "Information-theoretic analysis of an energy harvesting communication system," in Proc. 2010 IEEE Int. Symp. Personal, Indoor and Mobile Radio Communications Workshop, pp. 330-335.

[16] S. P. Boyd and L. Vandenberghe, Convex Optimization. Cambridge University Press, 2004.

[17] H. J. Prömel and A. Steger. The Steiner Tree Problem. Vieweg Verlag, 2002. 